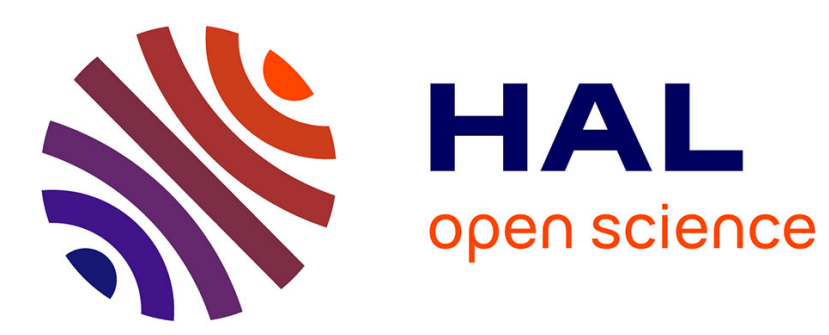

\title{
Travail informationnel et flux tendu
}

\author{
Jean-Pierre Durand
}

\section{To cite this version:}

Jean-Pierre Durand. Travail informationnel et flux tendu. Temps de travail et temps libre, 2000. hal-03582177

\section{HAL Id: hal-03582177 \\ https://hal.science/hal-03582177}

Submitted on 21 Feb 2022

HAL is a multi-disciplinary open access archive for the deposit and dissemination of scientific research documents, whether they are published or not. The documents may come from teaching and research institutions in France or abroad, or from public or private research centers.
L'archive ouverte pluridisciplinaire HAL, est destinée au dépôt et à la diffusion de documents scientifiques de niveau recherche, publiés ou non, émanant des établissements d'enseignement et de recherche français ou étrangers, des laboratoires publics ou privés. 


\title{
Travail informationnel et flux tendu
}

\author{
Jean-Pierre Durand
}

Le capitalisme a toujours été et restera nécessairement un système fondé sur l'économie du temps ; il est une économie du temps. Que ce soit du côté de la vitesse de rotation du capital ou du volume de valeur ajoutée par le travail d'un homme durant une unité de temps donnée, nous retrouvons toujours la question du temps (ce qui n'est pas vrai dans nombre d'autres systèmes économiques toujours en vigueur dans les interstices des sociétés africaines ou indiennes par exemple). La complexification de la production conduit à s'interroger sur les nouvelles façons d'accroître le résultat du travail des hommes (et des machines qu'ils manipulent) par unité de temps.

Le flux tendu (découvert "par accident" à partir du Just in time au Japon, dans l'automobile) est une réorganisation du travail qui, par définition (la bonne quantité de produits de bonne qualité au bon moment), lutte contre la flânerie des hommes et de la matière, c'est-à-dire contre la porosité du temps de travail (des hommes et des machines). Le flux tendu est par essence une réorganisation de la production de biens et de services (donc du travail) dont la référence principale est le temps ${ }^{1}$.

Parallèlement à la généralisation du flux tendu dans la production de biens puis dans les activités de service, nous avons assisté au développement de l'automatisation dans l'industrie et dans le tertiaire. Laquelle fait appel massivement aux technologies de l'information et de la communication (TIC) ; de même l'organisation technique du flux tendu recourt aux TIC. Or, les technologies de l'information, en tant que traitement automatique de l'information, ont déjà directement à voir avec le temps puisqu'elles ont considérablement accru les capacités de traitement de l'information par unité de temps, tout en réduisant les temps d'accès des hommes à ces mêmes informations. Ce n'est pourtant pas le thème que nous traiterons car il est probable que les gains de productivité en matière de traitement et de temps d'accès à l'information aient été en grande partie compensés par de nouvelles demandes colossales de traitement et de communication de l'information : complexification des processus de production et exigences accrues de qualité ou de réduction des délais (livraisons "en synchrone"). D'ailleurs les économistes ne parviennent pas à s'accorder sur l'importance des gains de productivité et sur

\footnotetext{
${ }^{1}$ Il est intéressant de noter qu'une des réorganisations du travail, qui couvre en partie le flux tendu, se dénomme time based management dans les pays anglo-saxons.
} 
l'efficacité économique des $\mathrm{TIC}^{2}$. En revanche, les TIC ont modifié les conditions d'exercice du travail des salariés, modifiant leur rapport à la matière dans l'industrie ou dans les services : la relation à l'objet de travail est plus ou moins médiatisée par l'outil informatique ou par tout autre outil de communication. Cette médiatisation est l'occasion d'une rationalisation à travers le travail sur les signes qui permettent d'accroître, dans certaines situations, l'activité productive par unité de temps considérée.

Alors, notre double questionnement peut s'exprimer ainsi : Quels sont les rapports entre technologies de l'information, temps et rationalisation du travail d'une part ? D'autre part, comment, malgré les différences objectives de contenus du travail, les différences de statuts, les différences de revenus, etc., le flux tendu unifie-t-il les conditions d'exercice du travail, en particulier parce qu'il gomme les différences de rapport au temps des catégories de salariés ?

\section{RAPPORT DU SALARIÉ À SON OBJET DE TRAVAIL, TRAVAIL SUR LES SIGNES}

Avant d'entrer dans le vif du sujet et au regard de la confusion conceptuelle régnant autour des technologies de l'information, il apparaît nécessaire de tenter une clarification rapide.

\section{Retour sur les concepts}

L'utilisation de plus en plus généralisée des technologies de l'information s'est accompagnée depuis trois décennies de tentatives de caractériser l'ère présente : société de service, ère postindustrielle, société informationnelle, etc. Si aucun de ces termes ne nous convient, puisqu'ils ne font pas référence aux modalités présentes d'accumulation du capital (avec les ruptures effectuées au tournant des années 80-90), il apparaît que les deux premières notions sont éminemment confuses.

Comme le rappelle opportunément M. Castells (1998), les activités manufacturières continuent non seulement de représenter une part importante du PNB américain (24\%) mais elles induisent directement les services liés à l'industrie (25\% du PNB). Si la notion de société post-industrielle veut signifier que les tâches subalternes, non qualifiées, répétitives et en travail posté disparaissent rapidement avec la réduction de la population ouvrière, la notion mystifie la réalité puisque ces mêmes tâches réapparaissent ailleurs dans le travail de bureau ou dans les services.

\footnotetext{
2 Cf. Pascal Petit, L'économie de l'information. Les enseignements des théories économiques, La Découverte, Paris, 1998 ou Timothy F. Bresnahan et al, Information, Technology, Workplace Organization and the demand for Skilled Labor : Firm-level evidence, Draft, MIT, 1998.
} 
A son tour, le terme de société de service, qui laisse entendre que nous vivons dans $L a$ Troisième vague (Toffler, 1980) après celle de l'agriculture et celle de l'industrie, masque toute l'importance de la diversité des activités de service. En effet, non seulement la nature du travail diffère selon les types de service, mais les technologies de l'information y jouent des rôles quelquefois opposés tandis que ces services occupent des fonctions radicalement différentes dans le renouvellement du mode d'accumulation du capital. Si l'on utilise les catégories de Singelmann (1978), elles-mêmes reprises par Delaunay et Cadrey (1987) et par Castells (1998), on peut distinguer :

- les services de distribution : transports, communications, commerces (tous réseaux)

- les services de production : banques, assurances, services aux entreprises, immobilier,

- les services sociaux : santé, éducation, services publics divers, qui relèvent de la consommation collective,

- les services personnels : hôtels, restauration, loisirs, personnel de maison, aides aux personnes (malades, personnes âgées), etc.

L'hétérogénéité des activités dans ces différentes catégories de service vaut bien celle propre à l'industrie, voire celle qui oppose les services à l'industrie ! C'est la raison pour laquelle il apparaît nécessaire de trouver d'autres entrées pour caractériser le travail dans le modèle productif émergent que celle qui distingue les secteurs primaire, secondaire et tertiaire (avec la prime de modernité accordée au dernier). La place qu'occupe l'activité dans les cycles de production et de circulation des marchandises et du capital a certainement moins d'importance aujourd'hui que le contenu de la tâche elle-même, en terme de création et de traitement de l'information.

Car l'un des grands changements intervenus récemment est bien celui de la pénétration massive des technologies de l'information et de la communication (TIC) dans l'espace de travail. D'où cette notion de révolution informationnelle (Lojkine, 1991) ou d'informationnalisme (Castells, 1998) pour marquer la rupture à laquelle nous assistons. Malheureusement, cette prime conceptuelle accordée aux technologies de l'information s'accompagne d'un angélisme surprenant : "Néanmoins le travail informationnel invite par nature à la coopération, au travail en équipe, à l'autonomie et à la responsabilité des travailleurs, sans quoi les nouvelles technologies ne peuvent fournir tout leur potentiel", écrit Castells (1998, 287). Comme quoi le déterminisme technologique ne peut mourir : les TIC possèdent ici naturellement la vertu de pousser à la coopération et à l'autonomie, ouvrant par là tout aussi tranquillement à l'épanouissement du salarié qui les utilise. Grâce aux vertus coopératives des 
$\mathrm{TIC}^{3}$, le capitalisme pourrait se dispenser d'effectuer un contrôle social sur le travail salarié. Si les TIC occupent le beau rôle dans la transformation des conditions de production de biens et de services, n'est-il pas préférable de comprendre comment elles renouvellent la division du travail, à la fois pour mobiliser les salariés et pour les mieux contrôler, y compris à travers leur propre implication?

\section{Technologies de l'information et renouvellement de la division du travail}

M. Castells (1998) a perçu la nécessité de repenser la division du travail et il nous propose une typologie à trois dimensions :

- la nature des tâches effectuées dans la création de valeur qui oppose les capitaines, les chercheurs, les concepteurs, etc. aux opérateurs et aux manoeuvres ;

- la relation du travailleur à son environnement qui oppose les connecteurs aux connectés et surtout aux déconnectés totalement rivés à leurs tâches ;

- la prise de décision qui oppose les décideurs aux exécutants en passant par les participants qui ne font que concourir à la prise de décision.

Pour descriptive et opérationnelle qu'elle soit, cette approche ne présente guère qu'une division technique du travail passant sous silence les nouvelles modalités du contrôle social sur le travail d'une part et la nature du rapport que les travailleurs entretiennent avec l'information selon leur place dans le système productif, d'autre part.

\section{La nature du travail et son contrôle}

Toute l'histoire du capitalisme a été celle d'une rationalisation du travail incluant un contrôle du travail effectué : salaires aux pièces pré-taylorien, norme de travail taylorienne, puis direction par objectifs pour les commerciaux généralisée par la suite à tous les cadres, etc. Or, le problème aujourd'hui posé est celui du contrôle social d'un travail de plus en plus intellectualisé, encore qu'il faille manier avec précaution cette notion. D'une part, parce que subsistent des millions de postes de travail dont la nature n'a pas été transformée et dans le coeur desquels les TIC n'ont pas pénétré. D'autre part, parce que cette intellectualisation recouvre plusieurs phénomènes qu'il faut différencier :

- le travail de conception occupe de plus en plus de salariés qui pensent les produits et les procédés car c'est au moment de leur conception que les gains futurs en qualité et en productivité sont les plus conséquents. De même, la conception des campagnes publicitaires,

\footnotetext{
3 Ou ailleurs, dans le même sens et en association avec les TIC, grâce aux nouvelles conditions de la concurrence (d'ailleurs liées aux TIC) qui exigent la réactivité et la créativité des travailleurs, le taylorisme ne peut que mourir laissant place à la nouvelle communication (Zarifian, 1997).
} 
des circuits commerciaux de plus en plus complexes, de leur logistique, etc. a créé des milliers de postes nouveaux dans les dernières décennies. Or, ce travail intellectuel de conception est difficile à normaliser et à mettre en procédure pour le contrôler : par nature, les démarches cognitives qui le sous-tendent sont imprévisibles et bien souvent différentes d'un individu à un autre. Pour ne prendre que l'exemple de la tentative de tayloriser la production des logiciels, celle-ci s'est soldée en général par de retentissants échecs.

- Le travail de surveillance des processus automatisés correspond à une intellectualisation du travail ouvrier (mais aussi du travail de bureau dans certains cas) : l'automatisation apparaît en effet comme une objectivation de l'intelligence humaine dans la machine, grâce aux technologies de l'information ; or, pour être surveillés et entretenus, ces systèmes intégrant plus de données et de procédures automatisés ("intelligence") exigent un niveau plus élevé d'intelligence humaine que le travail manuel direct d'hier. La question de la surveillance à moindre coût de travail humain, donc du contrôle de la qualité et de l'intensité du travail de surveillance, n'est pas une mince affaire. Si ce contrôle est plus aisé que le travail très intellectualisé de conception, il pose problème car il est avant tout une réponse à l'aléa qui par définition n'est guère prévisible, donc impossible à mette en procédure. D'où le choix radical de ne pas traiter l'aléa, mais plutôt d'empêcher son avènement par la maintenance préventive, laquelle peut être mise en procédures, offrant par là toutes les possibilités de contrôle d'un taylorisme amélioré (volume de travail fourni par unité de temps).

Il reste que l'aléa n'étant pas totalement évitable, le travail de surveillance demeure avec ses difficultés de contrôle social. Les salariés sont alors contrôlés non pas sur leur activité réelle mais sur leur aptitude à assurer un fonctionnement continu du système machinique : le contrôle ne porte plus sur l'activité humaine mais sur le résultat machinique induit par celle-ci par unité de temps. Le problème est que le lien entre les deux est régi par l'incertitude née de l'aléa propre au système machinique lui-même ; ainsi, les directions ont du mal à évaluer les besoins en main-d'oeuvre en matière de surveillance de processus automatisé. Face à l'incertitude du contrôle social du travail humain par le résultat machinique, elles introduisent peu à peu le contrôle sur la prévisibilité du comportement des salariés en cas d'aléa : lequel porte d'une part sur les aptitudes techniques (la justification) et d'autre part sur l'engagement et l'implication individuels (ou collectifs) face à l'aléa ; l'imbrication des deux définit largement la notion de compétence telle qu'elle est utilisée aujourd'hui dans les entreprises. Le contrôle social ne porte plus guère sur le travail fourni par unité de temps, mais sur le travailleur lui-même : c'est aussi le passage de la rémunération au poste à la rémunération fondée sur l'évaluation individuelle. 
- Enfin, le traitement des données, concomitant à la généralisation des TIC, peut donner lieu à un travail pas du tout intellectualisé : c'est le cas de la saisie de données pour leur traitement automatique (banques, assurances, facturation, dactylographie au kilomètre, INSEE...) ou celui du travail de caisse dans la grande distribution. Même s'il s'agit de travail faisant appel aux TIC, celui-ci ne porte que sur les signes qui ne font jamais sens, tandis que les qualifications mises en oeuvre restent faibles (lesquelles possèdent pourtant une importante dose de relationnel, par exemple chez les caissières). Dans ce travail de saisie de masse, le contrôle social du travail est resté très traditionnel, fondamentalement taylorien, à partir de la quantité et de la qualité du travail fourni (par exemple nombre d'articles passés en caisse à la minute, nombre d'erreurs, nombre de reprises, etc.). Mieux encore, les TIC sont utilisées directement, non pas pour soutenir ou pour remplacer un travail humain trop fastidieux et trop coûteux, mais pour le contrôler : c'est le terminal d'ordinateur ou la machine enregistreuse qui mesure l'efficacité du travail en temps réel et qui le communique à l'encadrement ${ }^{4}$.

Ainsi il n'y a pas de rapport de nécessité entre intellectualisation du travail et usage des TIC puisque certaines tâches recourant massivement aux TIC ne sont pas du tout intellectualisées. De même, il n'y a pas de lien nécessaire entre utilisation des TIC et accroissement de l'autonomie au travail. Enfin, nous avons pu relever une profonde ambivalence entre usage des TIC, intellectualisation du travail et développement de l'autonomie au travail dans le travail de surveillance des processus automatisés.

En effet, il ne saurait y avoir de rapport de nécessité entre ces trois pôles (usage des TIC, intellectualisation du travail, accroissement de l'autonomie), tout dépendant du rapport du salarié aux TIC et à travers celles-ci aux signes traités automatiquement, puis au signifié qu'ils véhiculent.

\section{Le sens du travail sur les signes}

Le développement des TIC et leur corollaire l'automatisation des procédés a eu pour conséquence de détacher toujours plus les ouvriers du contact direct avec la matière. C'est le principe de médiatisation du rapport à la matière par la machine automatique toujours plus performante, c'est-à-dire qui objective toujours plus de savoirs et d'intelligence humaine. Ce développement de l'automatisation, de la robotisation, de la "supervision" automatique, etc. a contribué à mieux marquer encore la différence entre travail direct sur la matière et travail indirect (de surveillance des machines, mais aussi d'encadrement, de conception, etc.).

\footnotetext{
4 Dans le télémarketing (ventes par téléphone), les procédures en temps réel sont les mêmes, affichant les scores des vendeurs en compétition, tandis que la maîtrise peut capter le ton des échanges pour les encourager ou pour les remercier.
} 
Parallèlement à cette tendance lourde (mais non absolue car il y a des tâches - de montage en particulier - qui sont trop coûteuses à automatiser) dans l'industrie, le développement des activités de service s'est accompagné d'une très forte croissance des emplois où le travail met les salariés en contact direct avec la matière : commerce de détail, restauration, hôtellerie, santé, etc. C'est la raison pour laquelle développement des TIC et automatisation ne signifient pas nécessairement réduction des activités en contact avec la matière. C'est aussi pourquoi à la distinction entre secteurs d'activité (agriculture, industrie, services) nous préférons celle qui distingue le travail direct avec la matière du travail indirect, car elle ouvre sur l'examen de la nature du travail réel, en particulier sur le rapport aux TIC des différentes catégories de salariés.

Du côté du travail indirect, les décideurs, les ingénieurs, les techniciens, les chefs de projet, l'encadrement, les ouvriers chargés de la surveillance des installations recourent aux TIC et doivent interpréter les signes qui leur sont fournis : c'est-à-dire qu'ils travaillent sur le sens des signifiants en les replaçant dans leur contexte afin d'initier une action : conception d'un produit ou d'un procédé, décision d'acheter ou de vendre, d'investir, etc., commandement d'un groupe ou directives à un individu, opération de chargement d'outil en dépannage avant le redémarrage... Pour ces salariés, la prise d'information sur un écran d'ordinateur (ou sur tout autre terminal des TIC) conduit à une mise en relation de cette information 5 avec d'autres informations, avec son contexte, avec autrui ; elle est bien travail sur le sens 6 .

C'est ici aussi la définition du travail relationnel tel qu'il est mis en évidence depuis deux décennies comme spécifiant le nouveau système productif. Toutes les catégories de salariés précitées - auxquelles on ajoutera le travail de surveillance des procédés tel qu'il est traité dans les pages ci-dessous - doivent mettre leur objet de travail en relation avec son environnement, ou doivent se mettre en relation avec des collègues pour atteindre les objectifs fixés : évidemment cette mise en relation passe par l'interprétation et des prises de sens des signifiants, c'est-à-dire par un va-et-vient des significations contextuelles des objets entre eux. Dans ce travail relationnel, on inclura aisément le travail d'encadrement : par définition, et même si ses

\footnotetext{
5 Il faudrait affiner ici ce processus - mais ce n'est pas le lieu - pour montrer comment l'information prise est incorporée dans l'acteur et comment c'est avec son corps et sa gestuelle qu'il va donner sens à l'information (au sens de la rendre efficace) en se mettant en relation avec autrui ou avec une autre machine porteur d'information, eux aussi . Cette incapacité signifie aussi que l'on ne peut dissocier les TIC des catégories d'espace et de temps : l'homme se meut, s'exprime et communique dans l'espace et dans le temps tandis qu'il n'y a pas d'information s'il n'y a pas, tôt ou tard, ici ou là, un humain susceptible de la recevoir pour l'interpréter. Sinon, l'information n'existe pas : elle est une somme de signes.

6 Ici, il ne s'agit pas de se méprendre sur la signification du sens : il ne s'agit pas du sens du travail dans l'aventure humaine en tant que valeur morale ou source de rédemption. Nous ne traitons ici que du sens - le signifié au sens des linguistes - des signes portés par la machine (ou par autrui) pour perpétuer le cycle de production (ou de distribution). La question n'est pas celle du sens du travail, mais de l'interprétation des signes pour atteindre l'objectif de production.
} 
objets différent, il est la mise en rapport, à travers la compréhension et l'interprétation, du cadre et de son environnement humain (celui qui le dirige comme ceux qu'il encadre).

Du côté du travail direct sur la matière (montage industriel, manutention dans la logistique, restauration, hôtellerie) mais aussi dans le travail de saisie de données (dactylographie, saisie informatique), le travail sur les signes ou ce que les organismes dénomment la prise d'information, ne s'accompagnent pas nécessairement d'un travail sur le sens de cette information, sur sa contextualisation et sur son interprétation, c'est-à-dire sur une réflexion conduisant à l'action. Bien au contraire, cette réflexion risque de nuire à l'efficacité du travailleur direct, essentiellement mesurée en terme de productivité apparente du travail. Par exemple, le monteur automobile lit un code sur la fiche suiveuse au cours d'un déplacement (durant son retour aux casiers en bordure de ligne par exemple) pour connaître le type de pièce à monter sur la voiture ; s'il s'interroge sur le numéro du casier, où le trouver, ou sur la fonction de la pièce ou sur la manière de le monter, il perd son temps et risque de "couler" sur la chaîne. Plus encore, pour empêcher toute réflexion et toute erreur, les organisateurs et les ingénieurs multiplient les poka-yoke ou détrompeurs : c'est dire que le signe - et de plus en plus le pictogramme sur les machines-outils - a bien plus d'importance que le signifié lui-même dans l'efficacité du travail. On peut dire la même chose autrement en montrant que cette prise d'information, sans préoccupation du contenu du signifié, correspond à une instrumentalisation de celui-ci. En parlant d'instrumentalisation du signifié nous voulons souligner que la prise d'information de l'ouvrier (ou de l'employé) sous forme d'un code ou de signes plus généralement, celui-ci va lui faire correspondre mentalement une procédure apprise par coeur. Laquelle a bien pour support un objet - le signifié - mais celui-ci ne fait pas appel à une réflexion et au sens qu'il peut prendre dans tel ou tel contexte. La procédure répétitive, comme moyen de gagner du temps - en tant qu'elle est rationalisation des gestes, institutionnellement ou non - fait appel au réflexe (antinomie de la réflexion) et aux automatismes. Autrement dit, dans nombre de postes et de fonctions recourant aux TIC, cet usage des TIC, quand il est instrumentalisation du signifié, c'est-à-dire primauté accordée au signe sur le signifié lui-même, ne garantit pas une élévation conséquente des qualifications en dehors de savoir lire et écrire 7 . Il n'y a donc pas de rapport univoque entre diffusion massive des TIC dans l'industrie et les ouvriers et accroissement des qualifications car nombre de postes de travail instrumentalisent le signifié sans rendre nécessaire le détour par le sens porté par les signes utilisés.

\footnotetext{
${ }^{7}$ Ce que confirment les statistiques relatives à l'illétrisme quand elles portent sur la compréhension de ce qui est lu.
} 
Entre ces deux pôles opposés du travail direct sur la matière instrumentalisant le signifié et le travail indirect recourant au sens des signifiants, se situe bien sûr une longue échelle sur laquelle varie ce que l'on pourrait dénommer le curseur de l'interprétation des signes : on trouvera dans les fonctions de conception des activités répétitives et procédurales sans détour par le sens des signes utilisés, tandis que les opérations codifiées dans la restauration rapide devront être interprétées en cas d'aléas (interprétation évidemment limitée à l'intérieur des possibles offerts par des procédures, en particulier sanitaires et/ou de rentabilité).

La surveillance des procédés de fabrication, de distribution ou de traitement de l'information se situe bien entre ces deux pôles. Par essence, l'aléa appelle une multiplicité de prises d'information (sur écran de surveillance, mais tout autant sur la machine elle-même), puis un diagnostic avant réparation et remise en route. En tant que démarche cognitive, le diagnostic nous parait fort intéressant puisque, malgré leurs efforts, ni les cogniticiens, ni les psychologues n'ont pu produire une codification générale qui permettrait de répondre à toutes les situations, c'est-à-dire l'importance de l'interprétation selon le contexte 8 et la nécessité de la réflexion. Mais en même temps, les directions tentent de codifier la démarche de diagnostic pour chaque situation donnée 9 à travers leur mise en procédure ou, comme on l'a déjà dit, de traiter préventivement les aléas ; laquelle maintenance préventive se fait en grande partie, elle aussi, par le développement des procédures. On pourrait montrer de même que les achats de biens ou de services, à l'intérieur de l'entreprise, ou les relations avec les clients (et avec les fournisseurs) font de plus en plus appel à des procédures extrêmement affinées : si la raison invoquée est la qualité du service, sa traçabilité et donc sa possible répétition, la préoccupation de réduction des coûts à travers l'utilisation des procédures apprises et utilisées automatiquement, restent le facteur dominant de leur justification.

Ainsi, il y a un lien étroit entre recherche de l'abaissement des coûts, généralisation des procédures y compris par l'usage des TIC et instrumentalisation du signifié : l'objectif est bien d'accroître l'intensité de l'activité productrice de valeur des salariés en évitant, quand c'est possible, le détour par la réflexion et l'interprétation ; la sortie du signe et sa transformation immédiate en action productrice de valeur, par réflexe ou automatisme appris, demeure un point essentiel de l'économie de temps qu'est le capitalisme.

\footnotetext{
8 Voir à ce propos les Limites de l'intelligence artificielle dans Hubert Dreyfus, Intelligence artificielle. Mythes et réalités, Flammarion, 1984.

9 C'est le sens de l'utilisation des systèmes experts qui permet justement de remplacer les experts par un personnel moins qualifié. Les résultats restent toutefois très en-deça des attentes.
} 


\section{FLUX TENDU ET DENSIFICATION DU TEMPS DE TRAVAIL}

Ici, la densification du temps signifie l'accroissement de l'intensité du travail, obtenue essentiellement par la réduction de la porosité du temps de travail : il ne s'agit pas d'accélération des rythmes de travail réel en tant que tel (on ne travaille pas plus vite car les "limites" de rapidité des gestes à qualité optimale sont déjà atteintes la plupart du temps 10), mais de l'abaissement des temps de récupération ou de la mise en activité des hommes en temps marqué par exemple (pendant que la machine travaille) : le résultat est bien une augmentation de l'activité productrice de valeur dans le temps imparti au travail.

Aujourd'hui, de plus en plus, la règle établie par les directions est de construire un flux continu de matière (ou d'informations, ou de consommateurs) dont les salariés sont responsables : toute rupture du flux est dénoncée comme coûteuse en temps puisque les salariés et les machines de l'aval ne travaillent plus ; la continuité du flux devient l'objectif premier de tous les salariés tandis que la règle de réduction des coûts (compétitivité par les coûts et discours sur la globalisation obligent) signifie que cette continuité du flux se fait à nombre de salariés minimal : cette deuxième règle organise à son tour la pression du temps sur les salariés qui doivent accélérer leur cadence d'intervention (ou allonger leur journée de travail pour les ingénieurs et les cadres ${ }^{11}$ ) afin de maintenir la continuité du flux.

Ce principe du flux tendu ne concerne plus seulement quelques industriels : de fait, il s'est généralisé à tous les secteurs industriels, mais aussi aux services : banque, assurance, grande distribution, tourisme, restauration rapide, etc. A bien y regarder, la relation "clientfournisseur", le management par projet, l'ingénierie simultanée empruntent largement au principe du flux tendu puisqu'à chaque fois il s'agit de livrer le plus rapidement possible une information ou un service de qualité à un client en aval.

Pour mener l'analyse du rapport entretenu par le flux tendu avec le temps, on peut considérer que les situations de travail, selon que le salarié surveille le flux, qu'il en est l'organisateur, ou bien que son rythme de travail est étroitement lié au flux lui-même. On verra ensuite que cette distinction analytique ne conduit pas toujours à une différence aussi essentielle des conditions de travail elles-mêmes.

\footnotetext{
10 Ces limites sont toujours relatives à un moment donné et à une population donnée (âge, résistance physique, dextérité, qualification, etc.) : l'idée de densification du temps de travail ne porte pas sur les cadences dans l'absolu mais dans leur relativité à la totalité du temps travaillé.

11 Quoique cet allongement de la durée du travail des cols blancs (accroissement extensif) diffère par nature de l'intensification du travail des autres salariés, on peut ici assimiler les deux phénomènes au sens où il s'agit d'accroître le temps productif de valeur par rapport au temps de travail rémunéré par l'employeur.
} 


\section{La place du temps dans la surveillance du flux}

Que ce soit dans la surveillance d'un flux productif (surveillance d'une ligne d'usinage ou d'un système informatique) ou dans l'organisation d'un flux sur lequel travaillent d'autres salariés (cadençage d'une ligne de montage automobile, queue de consommateurs à une caisse de grand magasin), les "gens du flux" ont pour objectif principal de ne pas rompre celui-ci. Ils ont intégré l'objectif qui ne se discute plus. Chacun s'oblige à tenir son rôle et à anticiper de façon à éviter tout aléa : l'implication sur cet objectif est "naturalisée" au sens où l'on a accepté, ou bien on quitte la fonction. Ce qui n'interdit pas les mécontentements ou les revendications quant au nombre de salariés nécessaires au maintien du flux : le débat sur les conditions et les charges de travail, face au souhait patronal de réduire les coûts, tourne autour du nombre d'interventions à effectuer par unité de temps ; il n'est pas rare de voir les ouvrier courir sur des lignes d'usinage automatisées afin de tenir l'objectif. Pour les ouvriers concernés - la question est de même nature pour les informaticiens chargés de maintenir un réseau et un système informatique - le travail est perçu comme une "lutte contre le temps" :

- en cas de rupture du flux, les postes de l'aval ne produisent plus et les gens du flux doivent le rétablir au plus vite,

- en temps normal, il faut accomplir les mesures préventives (changements d'outils, graissage...) en un minimum de temps et envisager de nouvelles actions de prévention des aléas.

La surveillance des flux productifs concentre une utilisation plus intensive des "ficelles", des "coups de main", des "secrets de fabrique", en un mot des qualifications tacites que les procédés de fabrication traditionnels : il y a urgence et les intéressés livrent plus facilement leurs savoirs individuels pour tenir l'objectif. Des techniques socio-productives comme la TPM (Total Productive Maintenance), les démarches qualité totale, le kaïzen (amélioration permanente) favorisent et encouragent la publicité des savoir-faire hier privés, leur partage entre ouvriers, voire leur appropriation par les services spécialisés concernés.

\section{La place du temps dans le travail à rythme imposé (flux)}

Dans l'ingénierie simultanée, chaque ingénieur ou chaque technicien doit livrer en temps utile un schéma, un projet, un ensemble de cotes (ou toute information dont la certitude n'est jamais absolue, contrairement au processus antérieur, séquentiel) à d'autres ingénieurs qui attendent ces informations pour continuer leur travail : tel est le principe du simultané, d'avoir des concepteurs en étroite dépendance les uns des autres pour réduire les coûts de conception, mais surtout pour penser des produits et des procédés plus "intelligents". Le résultat de cette 
dépendance mutuelle, ponctuée de réunions d'échanges d'informations, est la transformation de la fonction de conception en un vaste flux - non pas seulement linéaire mais plutôt brownien dans lequel chacun est contraint de livrer à autrui, dans les meilleurs délais, les informations les plus fiables : la pression du temps apparaît comme l'effet principal de l'ingénierie simultanée sur les conditions de travail.

Il en est de même avec le groupware, cette réunion à travers l'intranet des capacités de mémorisation et de traitement de l'informatique, qui permet à des dizaines de personnes de travailler ensemble sur le même projet de n'importe quelle nature qu'il soit. La dépendance réciproque des salariés, avec l'objectif temporel fixé par le chef de projet, contraint chacun à répondre dans les meilleurs délais à toutes les sollicitations de ses collègues. Sans lien physique de réunion, en dehors de toute organisation collective contrainte, chacun doit pourtant tenir compte de la collectivité virtuelle et organiser individuellement son temps de travail pour tenir les exigences temporelles de ses collègues. Les quelques recherches effectuées sur le groupware $^{12}$ concluent aux ressemblances organisationnelles avec le flux tendu et aux potentialités de contraintes temporelles sur les salariés que ce nouvel outil possède.

Du côté du travail des caissières de "grandes surfaces", le calcul de l'optimum du nombre de caissières est fait afin que chaque caissière subisse la pression de trois à six personnes dans la queue, selon la nature du magasin (bricolage, alimentation...) : au-delà, le magasin risque de voir les clients changer d'enseigne. Si l'organisation taylorienne calcule assez précisément le nombre d'articles à passer à la minute, la pression de la file d'attente conduit les caissières à tenir le rythme prévu sans que n'existe un encadrement de proximité très développé. La même file d'attente maintient la pression entre les clients, empêchant par là que la caissière s'aménage un espace de temps libre ${ }^{13}$.

Le travail de montage en chaîne d'appareils ménagers, d'appareils électroniques ou de voitures, reste bien sûr le cas d'école de la dépendance temporelle de chaque ouvrier par rapport au défilement du convoyeur. Et tous les aménagements ou modernisations actuels (ergonomie des casiers et des poste de travail, poka-yoké, servantes accompagnant le monteur, cabine le transportant au plus près de la voiture, etc.) ne visent qu'à accroître le temps productif (on parle de "postes efficaces"), c'est-à-dire à réduire encore un peu plus les temps grignotés durant les

12 Cf. Sylvie Craipeau et Béatrice Faguet-Picq, Le groupware: études monographiques, Evry, INT-CNET, 1997.

13 Dans le même sens et pour bien former (ou pour mettre en condition) les nouvelles caissières, elles sont systématiquement "oubliées" au moment de leur pause : la gestion de leur temps par autrui est la preuve irréversible de son aliénation au bénéfice de l'employeur. 
déplacements qui servaient jusqu'alors à la "récupération" du monteur face à la fatigue de l'acte productif $^{14}$.

On pourrait multiplier les analyses des mises en oeuvre du flux tendu pour montrer qu'il s'agit d'une perpétuation ou mieux encore d'un approfondissement du principe fordien du travail en continu et de l'exercice du contrôle social du travail par la contrainte du temps. Plus encore, le flux tendu en est l'extension au travail intellectuel, comme par exemple dans l'ingénierie simultanée ou dans le groupware. N'en déplaise aux panégyristes du nouveau dans l'entreprise, la compétitivité par les coûts demeure une priorité dans les entreprises et le flux tendu est un bon outil de contrôle social du travail effectif. Il peut réduire les coûts par l'organisation d'une pression du temps sur le salarié qui le conduit à exécuter le plus tôt possible la tâche impartie afin de ne pas rompre le flux. La pression du temps doit être comprise comme la résultante d'une double contrainte : celle de l'économie des moyens humains calculée par la direction ou le bureau des méthodes qui fixe le volume maximal acceptable. La tâche à accomplir par personne (ou par équipe) par unité de temps d'une part et celle de sa mise en oeuvre réelle, sous la responsabilité directe d'un chef qui impose l'exécution de la tâche dans le temps donné ou sous la contrainte de la non rupture du flux tendu, d'autre part.

\section{Contrainte du temps et contrainte au travail}

Le maintien de la continuité du flux tendu exige un travail collectif puisqu'il doit toujours y avoir une présence humaine pour surveiller l'état du flux et intervenir rapidement en cas d'aléa. D'où le travail en groupe de salariées polyvalentes et responsables collectivement de la continuité du flux. La polyvalence signifie aussi l'interchangeabilité des hommes que réclame la permanence de la présence humaine. Elle n'empêche pas non plus, paradoxalement, une certaine spécialisation de chaque membre de l'équipe, laquelle accroît par assemblage et par combinaison (plutôt que par additivité) la qualification collective de l'équipe ou du groupe. Les mêmes exigences du flux tendu entraînent une diffusion de la responsabilité dans le groupe de

14 Cf. J. Pierre Durand et al, L'avenir du travail à la chaîne, La Découverte, 1998, ou J. Pierre Durand et Nicolas Hatzfeld, La chaîne et le réseau,Ouvriers à Sochaux, à paraître. 
travail. Cette responsabilisation peut entraîner un stress ${ }^{15}$ lorsqu'elle est gérée a minima de

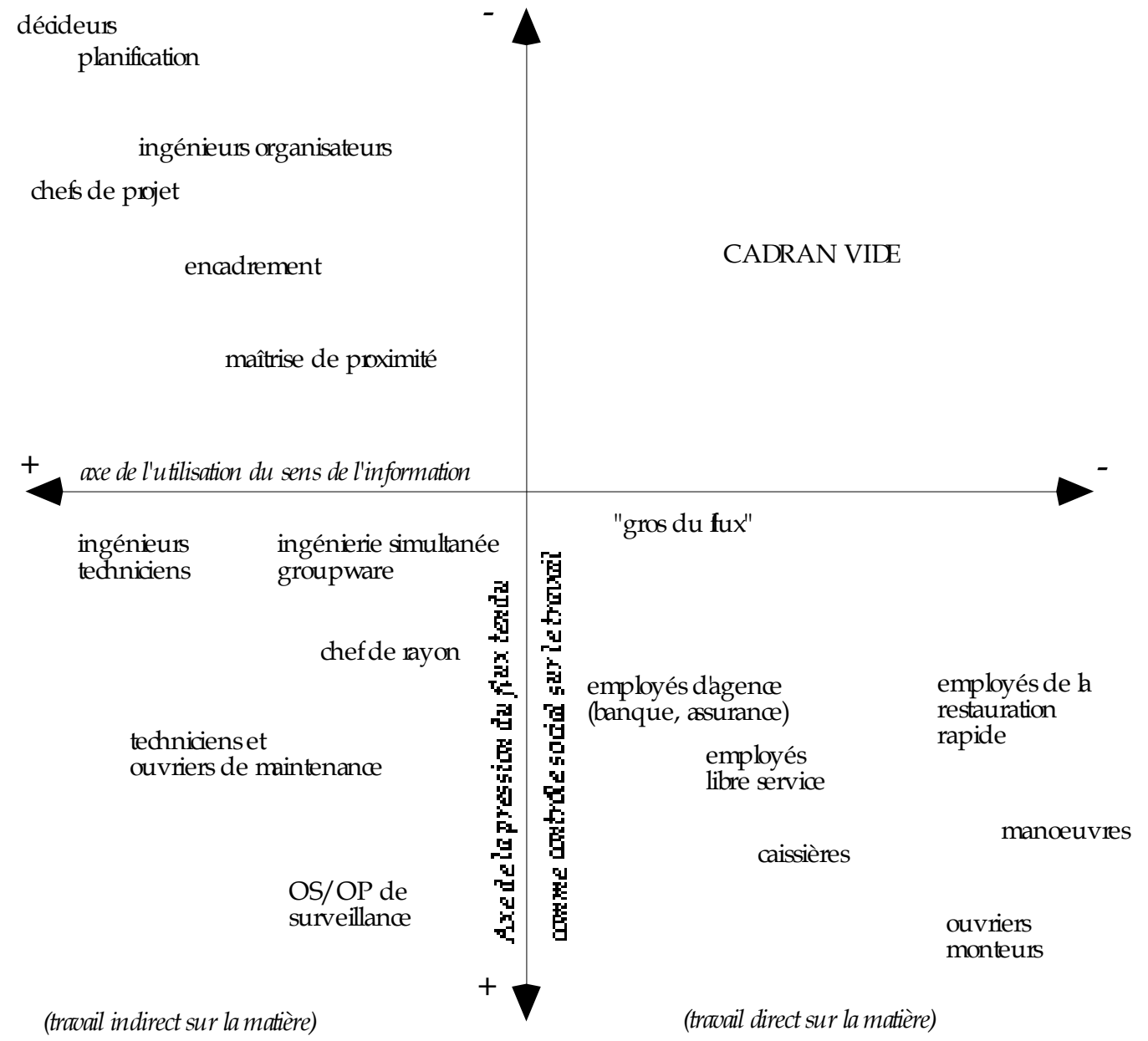

moyens humains, c'est-à-dire lorsque la continuité du flux, avec les tâches incompressibles qu'elle requiert, est prise en charge par une forme de travail quantitativement (voire qualitativement) insuffisante : c'est bien à nouveau la pression du temps, dans ces cas de réduction des coûts de main-d'oeuvre, qui occasionne le stress et dans certains cas sert d'outil de gestion (management par le stress).

Cette pression du temps et cette intériorisation de la continuité du flux rend superflue la présence d'un encadrement de proximité. D'où tous les grands programmes de raccourcissement de la ligne hiérarchique qui est un fondement bien plus objectif qu'il n'y parait : ces allégements des lignes hiérarchiques sont d'autant plus faciles à mettre en oeuvre que le flux tendu objective d'autres contraintes, d'une certaine manière plus radicales que la présence humaine du chef. La continuité du flux tendu réclame des interventions que le groupe de travail exécute "naturellement" pour tenir les objectifs qu'il a intériorisés. L'humanisation du management tient plus à cette "naturalisation" des contraintes continues dans le flux tendu qu'à une

15 Cf. Michael Parker and Jane Slaughter, Choosing sides : Unions and the Team Concept, a Labor Notes Book, Boston, South End Press, 1988. 
transformation culturelle des entreprises: la perception d'une plus grande autonomie des salariés, y compris face au temps, même si elle est illusoire, reste l'un des changements les plus importants de ces deux décennies ; le travail en groupe comme réponse organisationnelle cohérente avec les contraintes intrinsèques du flux tendu y est pour beaucoup.

Ces mêmes contraintes temporelles liées au flux tendu possèdent un autre effet moral et idéologique non négligeable : celui de mixer toutes les catégories de salariés (quelquefois dans de mêmes lieux) pour tenir les objectifs partagés par tous les acteurs. Chez un fabricant de pizza qui livre en 30 minutes, l'hôtesse d'accueil, le cuisinier et le livreur font équipe avec le manager pour que le délai soit tenu. Dans la surveillance d'une ligne d'usinage automatisée, les OS fabricants, les ouvriers de maintenance, les améliorateurs, le spécialiste TPM, les agents du bureau des méthodes, la maîtrise se rencontrent et débattent (presque) à même niveau des problèmes techniques rencontrés ayant perturbé le flux productif : malgré leurs divergences d'intérêts corporatifs (à la fois en matière de tableaux de bord, de primes, de rémunérations, de méthodes comptables, de renseignements du système informatique, etc.), les contraintes temporelles du flux tendu rassemblent toutes ces catégories professionnelles sur des objectifs partagés a minima.

En résumé, le flux tendu unifie les modalités du contrôle social sur le travail dans le groupe de travail ou avec son environnement. A travers l'objectivation ou la "naturalisation" des contraintes, le flux tendu masque la nature de ses objectifs en rapprochant les catégories de salariés malgré les différences de contenu du travail, la différence de statut, les différences de revenus, etc.

\section{TEChNOLOGIES DU SIGNE ET FLUX TENDU}

Après nous être interrogés sur les rapports entretenus par les technologies de l'information avec le temps, puis sur les rapports entretenus par la continuité du flux tendu avec le temps, nous pouvons croiser les résultats des analyses de ces deux objets pour construire une sorte de cartographie des postes de travail et des fonctions. L'axe des abscisses réfère à l'utilisation du sens porté par les signes traités par les TIC : les uns (décideurs, cadres, certains concepteurs...) ne travaillent que sur les signifiés, tandis qu'à l'opposé d'autres n'utilisent pas les TIC ou bien n'utilisent que des signifiés instrumentalisés ; ces derniers sont en général en contact direct avec la matière, dans l'industrie ou dans les services. L'axe des ordonnées réfère aux contraintes du flux tendu comme nouveau mode de contrôle social : il oppose les décideurs, les planificateurs 
(ceux qui donnent les objectifs du flux) à ceux dont l'activité est dominée par les impératifs du flux (postes dans le flux) en passant par ceux qui surveillent les flux productifs.

Il est possible de placer sur un tel espace la totalité des postes et des fonctions des différents secteurs d'activité. A partir du croisement des deux axes, il apparaît qu'il s'en constitue un troisième, virtuel, qui passe par la diagonale des cadrans 1 et 3 : il s'agirait d'un axe représentant à la fois l'autonomie dans le travail (maîtrise de son temps et de son organisation dans le travail) et l'intérêt porté au travail par les différentes catégories de salariés par rapport à leurs conditions de travail (pression ou non du flux...). A une extrémité se situeraient les décideurs, planificateurs, ingénieurs-organisateurs et à l'autre extrémité se trouveraient les ouvriers monteurs, les manoeuvres, les caissières, les employés de la grande distribution ou de la restauration rapide, etc. Logiquement, sur cet axe virtuel, les fonctions s'appuyant sur le signifié mobilisent l'intérêt du salarié dans l'exécution de son travail, tout en lui offrant une certaine autonomie puisqu'il vit peu sous la pression temporelle du flux. Il n'en est pas de même pour certaines fonctions et postes qui, tout en s'appuyant sur le sens de l'information, découragent les titulaires de diplômes en raison de l'utilisation trop massive des procédures et de l'existence des contraintes temporelles du flux : c'est le cas des ingénieurs et des techniciens d'exécution des projets ou du personnel diplômé chargé de la surveillance des flux productifs, souvent frustré par le travail routinier.

Enfin, il existe un cadran vide ou presque vide (on pourrait y mettre les postes de nettoyage des locaux, certains postes de manoeuvre ne faisant appel qu'au signifié instrumentalisé sans pour autant être contraints par un flux quelconque). Lequel peut nous conduire à douter de la pertinence du maintien des deux axes qui pourraient alors être réunis en un seul, par exemple l'axe virtuel décrit ci-dessus. Ce serait une erreur parce que disparaitrait alors la distinction des deux pôles (entre parenthèses dans la figure) du travail direct - ou indirect - sur la matière qui constitue un facteur discriminant dans l'analyse du contenu du travail. En effet, c'est ici la dimension par laquelle pénètre la nature du rapport au signe dans l'utilisation des TIC : travaille-t-on sur le sens de l'information ou seulement sur le signifié instrumentalisé ? Parallèlement, quelle est la part du travail dominé par les procédures dans la fonction occupée ?

On l'a compris, cette représentation à partir des deux axes ordonne les postes et les fonctions dans les secteurs d'activité à partir des deux fondements des transformations actuelles du travail, l'utilisation massive des technologies de l'information et la généralisation du flux tendu. Ces deux types d'outils conduisent à de nouvelles rationalisations du travail entendues comme un accroissement de l'efficacité du travail par unité de temps. Contrairement à ce qui est trop souvent écrit, les TIC s'accompagnent d'un renforcement des procédures en raison de 
l'efficacité et du contrôle social qu'elles portent en elles-mêmes ; de même le flux tendu, à travers la "naturalisation" des contraintes, prend le temps comme unité de compte puisque toute rupture du flux entraîne l'inactivité des autres acteurs du théâtre de la production. 


\section{BIBLIOGRAPHIE}

BRESNAHAN Timothy F. et al, Information, Technology, Workplace Organization and the demand for Skilled Labor : Firm-level evidence, Draft, MIT, 1998.

CASTELLS Manuel, La société en réseaux. L'ère de l'information, Paris, Fayard, 1998.

CRAIPEAU Sylvie et FAGUET-PICQ Béatrice, Le groupware: études monographiques, Evry, INT-CNET, 1997.

J. Pierre Durand et al, L'avenir du travail à la chaîne, La Découverte, 1998, ou J.Pierre Durand et Nicolas Hatzfeld, La chaîne et le réseau,Ouvriers à Sochaux, à paraître.

DELAUNAY Jean-Claude et GADREY Jean, Les enjeux de la Société de Service, Paris, PFNSP, 1987.

DURAND Jean-Pierre et al, L'avenir du travail à la chaîne, La Découverte, 1998.

DURAND Jean-Pierre, "Le nouveau modèle productif" in G. Bollier et C. Durand (coord.), La nouvelle division du travail, Paris, L'Atelier, 1999.

DURAND Jean-Pierre et HATZFELD, La chaîne et le réseau. Ouvriers à Sochaux, à paraître.

DREYFUS Hubert L., Intelligence artificielle. Mythes et réalités, Paris, Flammarion, 1984.

LOJKINE Jean, La révolution informationnelle, Paris, PUF, 1991.

PARKER Michael et SLAUGHTER Jane, Choosing sides : Unions and the Team Concept, a Labor Notes Book, Boston, South End Press, 1988.

PETIT Pascal, L'économie de l'information. Les enseignements des théories économiques, Paris, La Découverte, 1998.

SINGELMANN Joachim, The Transformation of Industry : From Agriculture to Service Employment, Beverly Hills, Sage, 1978.

TOFFLER Alain, La troisième vague, Paris, Denoël, 1980. 